\title{
Association between flat-panel computed tomography hyperattenuation and clinical outcome after successful recanalization by endovascular treatment
}

\author{
Jang-Hyun Baek, MD, ${ }^{1,2}$ Byung Moon Kim, MD, PhD, ${ }^{3}$ Ji Hoe Heo, MD, PhD, ${ }^{2}$ \\ Dong Joon Kim, MD, PhD, ${ }^{3}$ Hyo Suk Nam, MD, PhD, ${ }^{2}$ Young Dae Kim, MD, PhD, ${ }^{2}$ \\ Hyun Seok Choi, MD, ${ }^{3}$ Jun-Hwee Kim, MD, ${ }^{3}$ and Jin Woo Kim, MD ${ }^{4}$
}

\begin{abstract}
${ }^{1}$ Department of Neurology, Kangbuk Samsung Hospital, Sungkyunkwan University School of Medicine, Seoul; ${ }^{2}$ Department of Neurology, Severance Stroke Center, Severance Hospital, Yonsei University College of Medicine, Seoul; ${ }^{3}$ nterventional Neuroradiology, Severance Stroke Center, Severance Hospital, and Department of Radiology, Yonsei University College of Medicine, Seoul; and "Department of Radiology, Gangnam Severance Hospital, Yonsei University College of Medicine, Seoul, Republic of Korea
\end{abstract}

OBJECTIVE Hyperattenuation on CT scanning performed immediately after endovascular treatment (EVT) is known to be associated with the final infarct. As flat-panel CT (FPCT) scanning is readily accessible within their angiography suite, the authors evaluated the ability of the extent of hyperattenuation on FPCT to predict clinical outcomes after EVT.

METHODS Patients with successful recanalization (modified Thrombolysis in Cerebral Infarction grade $2 \mathrm{~b}$ or 3 ) were reviewed retrospectively. The extent of hyperattenuation was assessed by the Alberta Stroke Program Early CT Score on FPCT (FPCT-ASPECTS). FPCT-ASPECTS findings were compared according to functional outcome and malignant infarction. The predictive power of the FPCT-ASPECTS with initial CT images before EVT (CT-ASPECTS) and follow-up diffusion-weighted images (MR-ASPECTS) was also compared.

RESULTS A total of 235 patients were included. All patients were treated with mechanical thrombectomy, and $45.5 \%$ of the patients received intravenous tissue plasminogen activator. The mean $( \pm S D)$ time from stroke onset to recanalization was $383 \pm 290$ minutes. The FPCT-ASPECTS was significantly different between patients with a favorable outcome and those without (mean $9.3 \pm 0.9$ vs $6.7 \pm 2.6$ ) and between patients with malignant infarction and those without ( $3.4 \pm$ 2.9 vs $8.8 \pm 1.4$ ). The FPCT-ASPECTS was an independent factor for a favorable outcome (adjusted OR $3.28,95 \% \mathrm{Cl}$ $2.12-5.01$ ) and malignant infarction (adjusted OR $0.42,95 \% \mathrm{CI} 0.31-0.57$ ). The area under the curve (AUC) of the FPCTASPECTS for a favorable outcome $(0.862$, cutoff $\geq 8)$ was significantly greater than that of the CT-ASPECTS $(0.637)$ ( $p$ $<0.001)$ and comparable to that of the MR-ASPECTS $(0.853)(p=0.983)$. For malignant infarction, the FPCT-ASPECTS was also more predictive than the CT-ASPECTS (AUC 0.906 vs $0.552 ; p=0.001$ ) with a cutoff of $\leq 5$.

CONCLUSIONS The FPCT-ASPECTS was highly predictive of clinical outcomes in patients with successful recanalization. FPCT could be a practical method to immediately predict clinical outcomes and thereby aid in acute management after EVT.

https://thejns.org/doi/abs/10.3171/2020.7.JNS193214

KEYWORDS endovascular treatment; stroke; prognosis; flat-panel computed tomography; vascular disorders

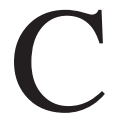
ONVENTIONAL multidetector CT is often performed after endovascular treatment (EVT) of acute stroke, as CT imaging can show periprocedural hemorrhagic conditions, including procedure-related intracranial hemorrhage and immediate hemorrhagic transformation due to reperfusion. However, most hyperattenuation on CT scanning is generally contributed by the contrast medium, so-called contrast staining. ${ }^{1-5}$ This contrast material-related hyperattenuation is thought to result from leakage of contrast medium into the parenchymal extracellular space due to blood-brain barrier disruption caused by focal ischemic injury, chemotoxicity, or reperfusion injury. ${ }^{6}$ Therefore, hyperattenuation due to contrast staining on postprocedural CT can be presumed to reflect the infarct core. ${ }^{1,2}$

ABBREVIATIONS ASPECTS = Alberta Stroke Program Early CT Score; AUC = area under the ROC curve; EVT = endovascular treatment; FPCT = flat-panel CT; ICA = internal carotid artery; $\mathrm{mRS}=$ modified Rankin Scale; NIHSS = National Institutes of Health Stroke Scale; ROC = receiver operating characteristic; tPA = tissue plasminogen activator.

SUBMITTED November 27, 2019. ACCEPTED July 15, 2020.

INCLUDE WHEN CITING Published online December 25, 2020; DOI: 10.3171/2020.7.JNS193214. 
CT-like images can be obtained directly within the angiography suite with the use of modern angiographic systems equipped with a flat-panel detector (flat-panel CT [FPCT]). FPCT is often performed during or at the end of the EVT procedure, primarily to observe periprocedural hemorrhagic conditions, due to the ease with which it can be performed and its recently improved image quality. Given that hyperattenuation on postprocedural CT is associated with the infarct core, we reasoned that the extent of hyperattenuation on FPCT performed at the end of the EVT procedure might be closely associated with patients' clinical outcomes. In particular, we hypothesized that the greater the extent of hyperattenuation on FPCT, the poorer the patients' clinical outcomes. To test this hypothesis, we evaluated clinical outcomes according to the extent of hyperattenuation of FPCT and compared the ability of FPCT to predict clinical outcome with that of other imaging modalities.

\section{Methods \\ Participants}

We retrospectively reviewed consecutive acute stroke patients with intracranial large-vessel occlusion of the anterior circulation who underwent EVT between January 2010 and December 2018. Patients were selected from a prospectively maintained stroke registry at a tertiary stroke center. EVT was generally considered for patients who met the following criteria: 1) a CT angiography-determined endovascularly accessible intracranial largevessel occlusion associated with neurological symptoms; 2) presentation to the hospital within 8 hours from stroke onset (in the later study period, patients within 8 to 12 hours were also considered if they had an Alberta Stroke Program Early CT Score [ASPECTS] $\geq 7$ ); and 3) baseline National Institutes of Health Stroke Scale (NIHSS) score $\geq 4$. We also generally performed EVT in patients with premorbid modified Rankin Scale (mRS) scores $\leq 3$. The intracranial internal carotid artery (ICA) and $\mathrm{M}_{1}$ or proximal $\mathrm{M}_{2}$ segment of the middle cerebral artery were defined as intracranial large vessels. In the present study, patients 1) whose occlusion was successfully recanalized and 2) who underwent FPCT at the end of the EVT procedure were included.

All patients eligible for intravenous tissue plasminogen activator (tPA) or EVT were evaluated by noncontrast CT and CT angiography. Patients eligible for intravenous tPA treatment were treated with $0.9 \mathrm{mg} / \mathrm{kg} \mathrm{tPA}$. EVT procedures were performed according to common recommendations. The frontline EVT modality was stent retriever thrombectomy, contact aspiration thrombectomy, or a combination of these techniques. A balloon-guiding catheter was routinely used. Successful recanalization in EVT was defined as a modified Thrombolysis in Cerebral Infarction grade of $2 \mathrm{~b}$ or 3 . Follow-up MRI, including diffusion-weighted imaging and MR angiography, were performed routinely at around 24 hours after the EVT procedure.

The institutional review board approved this study and waived the requirement of informed consent for study inclusion based on its retrospective design.

\section{Acquisition of FPCT}

FPCT was routinely performed at the end of the EVT procedure to evaluate if procedure-related complications (e.g., subarachnoid hemorrhage) or hemorrhagic transformation had occurred. In patients who underwent intracranial stenting or received intraarterial glycoprotein IIb/IIIa inhibitor, FPCT was performed before the introduction of rescue modalities to evaluate the hemorrhagic condition. FPCT images were acquired on a biplane angiography system (Allura Xper FD20/20 or Allura Clarity FD20/20 Interventional Angiographic Systems, Philips Healthcare). This imaging system allows motorized rotational movement of the C-arm in the plane perpendicular to the longitudinal table axis around the head of the patient's position at the system isocenter. A total of 620 fluoroscopic frames in a circular motion were acquired with the following parameters: voltage $120 \mathrm{kV}$, tube current $250 \mathrm{~mA}$, frame speed 30 frames per second, acquisition time $5 \mathrm{msec}$ per frame, total angle $240^{\circ}$, rotation speed $22^{\circ}$ per second, exposure time 20 seconds, field of view $48 \mathrm{~cm}$, and 1024 $\times$ 1024-pixel matrix detector. Image reconstruction was performed using a dedicated workstation. Image postprocessing to correct for beam hardening and scatter was performed to achieve maximum spatial and contrast resolution. For evaluation, volume-rendering reconstruction was performed with 5-mm-thick slices and no gap, although the thickness can be customized from 1 to $100 \mathrm{~mm}$.

\section{Determination of FPCT-ASPECTS, CT-ASPECTS, and MR-ASPECTS}

The extent of FPCT hyperattenuation was semiquantitatively assessed using the ASPECTS (FPCT- ASPECTS). To compare the predictive power of the FPCT-ASPECTS with other periprocedural imaging modalities, the ASPECTS on noncontrast CT images obtained before the EVT procedure (CT-ASPECTS) and diffusion-weighted images obtained after the EVT procedure (MR-ASPECTS) were also assessed. The CT-ASPECTS was calculated according to the original method.? Similarly, the ASPECTS was calculated by subtracting the number of hyperattenuated regions from 10 to calculate the FPCT-ASPECTS and MR-ASPECTS. Locations of interest for determining the FPCT-ASPECTS and MR-ASPECTS were the same as those for the CT-ASPECTS. The CT-ASPECTS, FPCTASPECTS, and MR-ASPECTS were assessed by a total of six independent neuroradiologists and interventional neuroradiologists. The ASPECTS on each imaging modality was assessed independently by two raters. All raters were blinded to clinical and procedural outcomes. Interobserver agreements of the CT-ASPECTS, FPCT-ASPECTS, and MR-ASPECTS were $0.872,0.936$, and 0.957 , respectively. Cases with discrepancies were resolved by consensus.

\section{Statistical Analysis}

To determine if there was an association between the FPCT-ASPECTS and clinical outcome after EVT, patients were grouped according to clinical outcome. In the present study, clinical outcome was evaluated based on functional outcome and malignant infarction.

Functional outcome was considered favorable if the 
mRS score at 3 months after EVT was $\leq 2$. Based on this threshold, patients were assigned to the favorable outcome or the unfavorable outcome group. First, demographic factors, common risk factors for stroke, clinical and procedural information, and the FPCT-ASPECTS were compared between groups. The Student t-test, Mann-Whitney U-test, chi-square test, and Fisher's exact test were used for comparison. To identify independent variables associated with a favorable outcome, multivariable binary logistic regression analysis was performed. Variables with $\mathrm{p}<0.10$ in univariable analysis were entered into the multivariable model. Second, to determine the predictive power of the FPCT-ASPECTS for a favorable outcome, we performed receiver operating characteristic (ROC) curve analysis and calculated the area under the ROC curve (AUC) of the FPCT-ASPECTS for a favorable outcome. An optimal cutoff point of the FPCT-ASPECTS for a favorable outcome was determined by the Youden index. Then, using DeLong's test, the AUC of the FPCT-ASPECTS for a favorable outcome was compared with those of the CTASPECTS and MR-ASPECTS.

The association of the FPCT-ASPECTS with malignant infarction was assessed using the same statistical approach as described above. For malignant infarction, patients were assigned to the malignant infarction group or the no malignant infarction group. Malignant infarction was defined as brain swelling that was treated with decompression craniectomy or that caused death without intracerebral hemorrhage. Brain swelling was assessed on MR images routinely obtained around 24 hours after the EVT procedure. If a patient's neurological status worsened, follow-up CT or MRI was performed immediately. Additional brain imaging was often performed serially for high-risk patients to observe the evolution of brain swelling. All statistical analyses were performed using SPSS (version 23, IBM Corp.) and MedCalc (version 16.8.4) software; $\mathrm{p}<0.05$ was considered statistically significant.

\section{Results}

A total of 279 patients had both a successful recanalization and FPCT during the study period. FPCT images could be assessed in 235 patients (mean age $68.1 \pm 13.6$ years; 50.2\% male) (Fig. 1).

\section{FPCT-ASPECTS and a Favorable Outcome}

After excluding 16 patients whose 3-month mRS scores were not available, 219 patients were analyzed (Fig. 1). Among them, 157 (71.7\%) were classified as having a favorable outcome. The mean FPCT-ASPECTS was significantly higher in patients with a favorable outcome $(9.3 \pm$ $0.9)$ than in those with an unfavorable outcome (6.7 \pm 2.6$)$ ( $<<0.001$; Table 1 and Fig. 2). Along with a lower NIHSS score, the FPCT-ASPECTS was an independent factor for a favorable outcome (OR 3.28, 95\% CI 2.15-5.01; p $<0.001)$. The AUC of the FPCT-ASPECTS for a favorable outcome was 0.862 (95\% CI 0.809-0.905; p < 0.001), which was significantly greater than that of the CT-ASPECTS $(0.637 ; \mathrm{p}<0.001)$ and similar to that of the MRASPECTS (0.853; $p=0.983$; Fig. 3A). An optimal cutoff

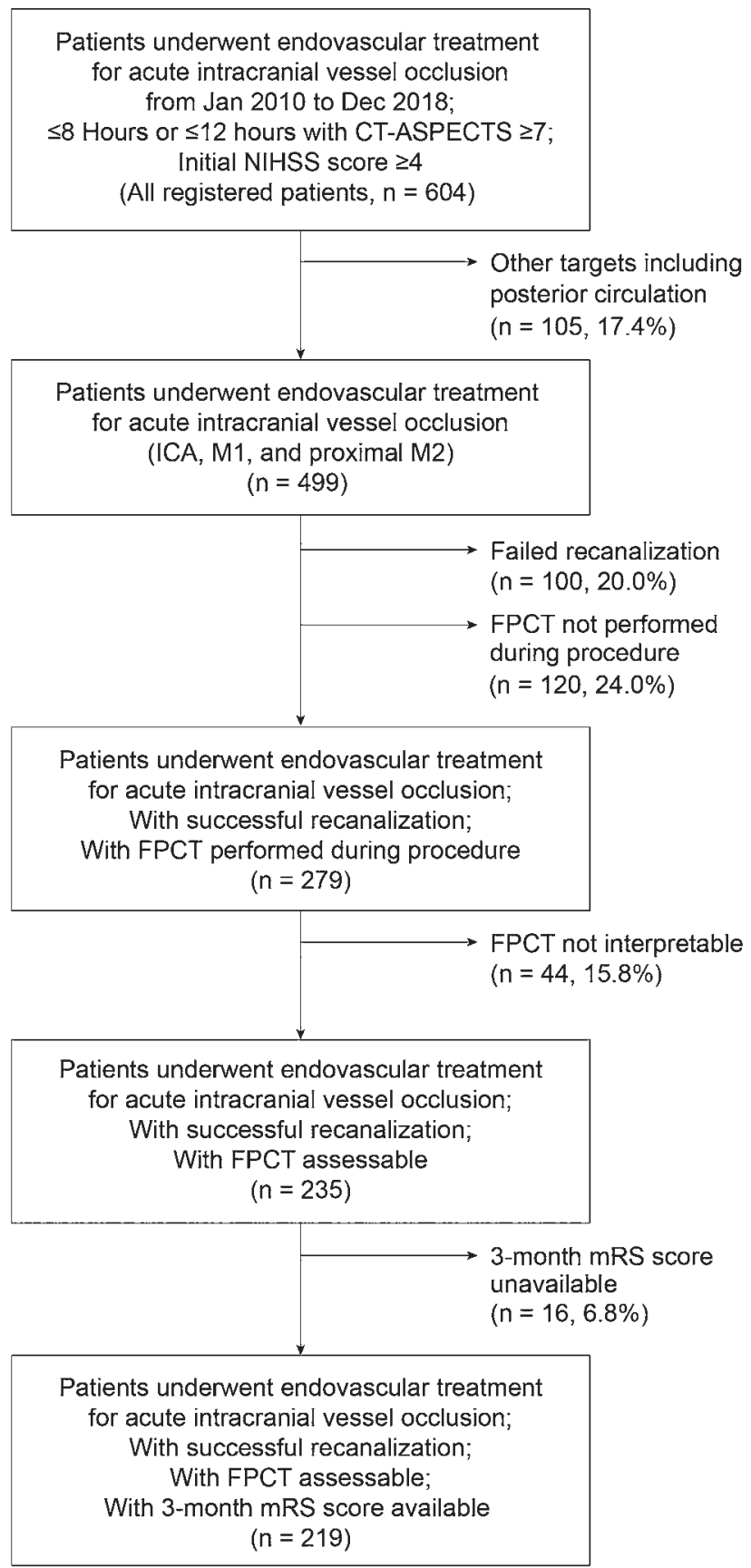

FIG. 1. Patient selection flowchart.

point of the FPCT-ASPECTS for a favorable outcome was $\geq 8$ (sensitivity $96.8 \%$, specificity $54.8 \%$ ).

\section{FPCT-ASPECTS and Malignant Infarction}

The mean FPCT-ASPECTS was significantly lower in patients with malignant infarction $(3.4 \pm 2.9)$ than in patients without malignant infarction $(8.8 \pm 1.4)(\mathrm{p}<0.001$; Table 2). Furthermore, the FPCT-ASPECTS was an independent factor for malignant infarction (OR $0.42,95 \% \mathrm{CI}$ $0.31-0.57 ; \mathrm{p}<0.001)$. The AUC of the FPCT-ASPECTS for malignant infarction was 0.906 (95\% CI 0.861-0.940; 
TABLE 1. Comparison of variables and FPCT-ASPECTS between patients with and those without a favorable outcome

\begin{tabular}{|c|c|c|c|c|c|c|}
\hline & \multicolumn{4}{|c|}{ Univariable Analysis } & \multicolumn{2}{|c|}{ Multivariable Analysis } \\
\hline & $\begin{array}{l}\text { All Patients } \\
(n=219)\end{array}$ & $\begin{array}{l}\text { Favorable Outcome } \\
\qquad(n=157)\end{array}$ & $\begin{array}{l}\text { Unfavorable Outcome } \\
\qquad(n=62)\end{array}$ & p Value & $\begin{array}{c}\text { OR } \\
(95 \% \mathrm{Cl})^{*}\end{array}$ & $\mathrm{p}$ Value \\
\hline Age, yrs & $68.1 \pm 13.7$ & $66.0 \pm 13.9$ & $73.1 \pm 11.7$ & $<0.001$ & $0.96(0.92-1.00)$ & 0.057 \\
\hline Male sex & $112(51.1)$ & $84(53.5)$ & $28(45.2)$ & 0.295 & & \\
\hline Hypertension & $121(55.3)$ & $88(56.1)$ & $33(53.2)$ & 0.764 & & \\
\hline Diabetes & $39(17.8)$ & $25(15.9)$ & $14(22.6)$ & 0.246 & & \\
\hline Smoking & $26(11.9)$ & $21(13.4)$ & $5(8.1)$ & 0.356 & & \\
\hline Atrial fibrillation & $86(39.3)$ & $56(35.7)$ & $30(48.4)$ & 0.092 & $0.67(0.28-1.59)$ & 0.363 \\
\hline Coronary artery disease & $26(11.9)$ & $19(12.1)$ & $7(11.3)$ & 0.999 & & \\
\hline Initial NIHSS score & $15.0[7.0,23.0]$ & $14.0[6.7,21.3]$ & $18.0[12.0,24.0]$ & $<0.001$ & $0.83(0.75-0.93)$ & 0.001 \\
\hline Intravenous tPA & $102(46.6)$ & $74(47.1)$ & $28(45.2)$ & 0.881 & & \\
\hline ICA occlusion & $85(38.8)$ & $54(34.4)$ & $31(50.0)$ & 0.045 & $0.92(0.36-2.32)$ & 0.851 \\
\hline \multicolumn{7}{|l|}{ Time profile, mins } \\
\hline Onset-to-image & $238 \pm 284$ & $232 \pm 465$ & $251 \pm 288$ & 0.664 & & \\
\hline Image-to-puncture & $89 \pm 67$ & $90 \pm 73$ & $87 \pm 43$ & 0.807 & & \\
\hline Puncture-to-recanalization & $52 \pm 38$ & $46 \pm 34$ & $67 \pm 42$ & $<0.001$ & $0.99(0.98-1.01)$ & 0.533 \\
\hline Onset-to-recanalization & $380 \pm 298$ & $368 \pm 299$ & $406 \pm 294$ & 0.399 & & \\
\hline CT-ASPECTS & $9.3 \pm 1.1$ & $9.5 \pm 0.9$ & $8.9 \pm 1.4$ & 0.001 & $1.33(0.90-1.97)$ & 0.157 \\
\hline FPCT-ASPECTS & $9.0 \pm 2.0$ & $9.3 \pm 0.9$ & $6.7 \pm 2.6$ & $<0.001$ & $3.28(2.15-5.01)$ & $<0.001$ \\
\hline
\end{tabular}

Univariate results are expressed as mean $\pm S D$, the number of patients (\%), or median [IQR].

* Odds ratio for a favorable outcome.

$\mathrm{p}<0.001$ ) with an optimal cutoff point of $\leq 5$ (sensitivity $82.4 \%$, specificity $97.3 \%$ ). The AUC of the FPCTASPECTS was significantly greater than that of the CTASPECTS (0.552; $p=0.001 ;$ Fig. 3B).

\section{Discussion}

In this study, we found that the FPCT-ASPECTS was significantly associated with favorable outcome and malignant infarction in patients with successful recanalization by EVT. More importantly, the FPCT-ASPECTS was significantly more predictive of a favorable outcome and malignant infarction than the CT-ASPECTS. The FPCTASPECTS was comparable to the MR-ASPECTS with regard to prediction of a favorable outcome. To the best of our knowledge, this is the first study of hyperattenuation on FPCT focusing on patients' clinical outcomes and the predictive ability of FPCT; most previous studies have focused on the association between hyperattenuation on imaging and hemorrhagic risk.

\section{Hyperattenuation on Postprocedural CT and Clinical Prognosis}

Early prediction of a patient's clinical prognosis is important in acute stroke management. Physicians can make short- or long-term management plans and monitor possible detrimental clinical events based on the prediction results. In routine clinical practice, malignant infarctions develop in the acute period, and later functional outcomes require prediction. Clinical outcomes after EVT have been predicted using common prognostic factors such as stroke extent based on initial brain images, time from stroke onset to recanalization, and a patient's collateral status, to mention a few factors. ${ }^{6,8-11}$ Although many factors are well known to be associated with clinical outcomes after EVT, it is not easy to draw a conclusion by integrating these factors. Successful recanalization is also a strong determinant of a patient's clinical outcome. Nowadays, most patients have a successful recanalization with advances in endovascular technique. However, despite the successful recanalization, a favorable outcome cannot be achieved in a considerable number of patients. Thus, something more direct and practical is required to immediately predict clinical prognosis in patients with a successful recanalization.

Postprocedural CT findings may be useful for predicting a patient's clinical prognosis. Conventional CT is primarily performed after an EVT procedure to observe hemorrhagic conditions such as procedure-related intracranial hemorrhage or hemorrhagic transformation. Furthermore, hyperattenuation on postprocedural conventional CT images is a known risk factor for subsequent hemorrhagic transformation. ${ }^{3,12-14}$ According to previous studies, a substantial portion of hyperattenuation is due to contrast staining, not hemorrhage. Contrast staining on postprocedural conventional CT results from blood-brain barrier disruption, which is in turn caused mostly by ischemic injury of the affected region. In other words, regions of contrast staining on postprocedural CT indicate the extent of blood-brain barrier disruption and may be a surrogate of the final infarct core volume or infarction area. In support of this, the extent of hyperattenuation on postprocedural conventional CT images has been shown to match the final infarct volume well., ${ }^{1,2}$ FPCT is readily assessable and is widely performed during or at the 


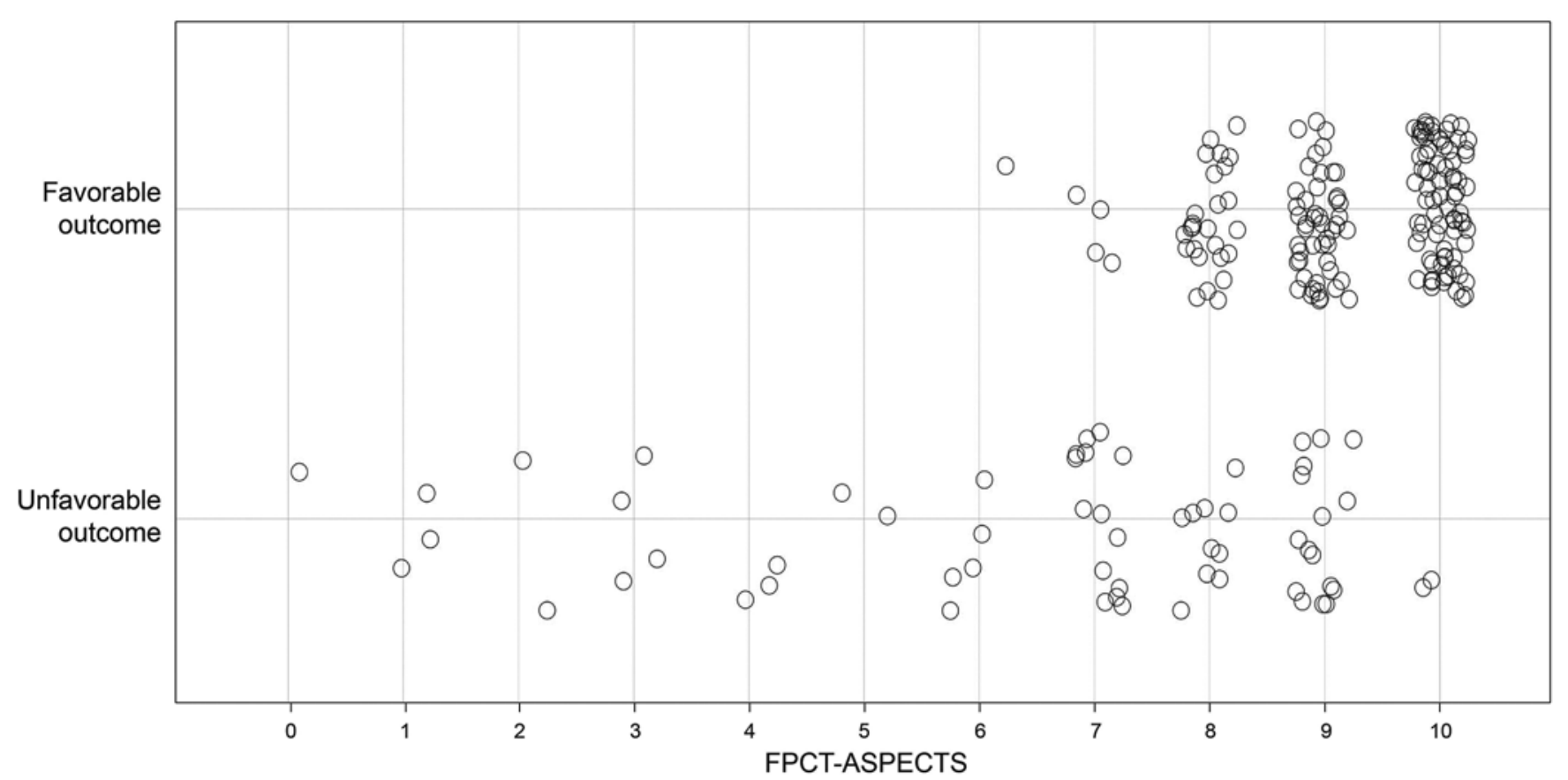

FIG. 2. Distribution of functional outcome according to the ASPECTS on FPCT (FPCT-ASPECTS).

end of endovascular procedures for the same purposes as postprocedural conventional CT. FPCT can detect postprocedural hemorrhagic conditions equally as well as conventional CT. ${ }^{15,16}$ The extent of hyperattenuation on FPCT is also associated with subsequent hemorrhagic transformation and final infarct volume. ${ }^{17,18}$ If hyperattenuation on conventional CT or FPCT is significantly associated with final infarct volume, the extent of hyperattenuation may be predictive of a patient's clinical prognosis.

\section{Advantages of Using FPCT After EVT}

In addition to its clinical relevance, FPCT has several advantages over conventional CT. FPCT is easier to perform; it can be performed on the same angiography table without transferring the patient to the CT room. Although the burden in transporting a patient to the $\mathrm{CT}$ room has been reduced by the recent availability of a portable CT scanner, FPCT is still more convenient. Furthermore, FPCT can be repeated at any time during the endovascular procedure. For this reason, FPCT performed at the end of the EVT procedure is starting to replace conventional CT performed after the EVT procedure. In addition, by performing FPCT at the end of the EVT procedure, FPCT might show hyperattenuation more optimally. Because hyperattenuation on CT might attenuate even over a short period of time, the timing of CT scanning is important. Even on conventional CT scanning performed approximately 1 hour after the EVT procedure, a considerable area of the infarction can become iso- or hypoattenuated. ${ }^{19}$ However, our findings in this study do not mean that FPCT is the only CT modality to show the predictability of clinical outcomes after EVT. If postprocedural CT findings need to be evaluated, any type of CT scanner can be considered.
We think that the postprocedural findings from FPCT could be also applied to findings from a conventional or portable CT scanner.

FPCT can be practically useful in a few ways. First, by performing FPCT at the end of the EVT procedure, procedure-related intracranial hemorrhage can be evaluated. Hemorrhagic transformation that develops during or as a result of EVT can also be observed. In cases in which cervical carotid stenting, intracranial stenting, or glycoprotein $\mathrm{IIb} / \mathrm{III}$ infusion is required, hemorrhagic conditions can easily be detected by performing FPCT during the EVT procedure. Although clinical or neurological examination findings could also hint at intracranial hemorrhage, neurological deterioration might possibly be due to other conditions, such as the progression of ischemic stroke, metabolic derangement, and unstable vital signs. In addition, unrecognized intracranial hemorrhage should be found in patients who need immediate antithrombotic medications after or during EVT. Second, by evaluating hyperattenuation on FPCT at the end of the EVT procedure, a patient's functional outcome, in addition to the possibility of malignant infarction, can be predicted at an earlier stage. This could help physicians monitor and prepare for further detrimental clinical events. With this clinical utilization, our center has performed FPCT in cases of EVT. After every procedure, we perform FPCT scanning rather than CT scanning.

Although the AUC of the FPCT-ASPECTS in predicting patient's functional outcome is similar to that of the MR-ASPECTS, we do not think it directly means that MR images obtained after EVT can be replaced with FPCT. Generally, MR images have their own role in acute stroke management: to define the exact extent of acute stroke and involved anatomical structures, to detect hemorrhagic transformation more sensitively, and to evaluate the follow- 

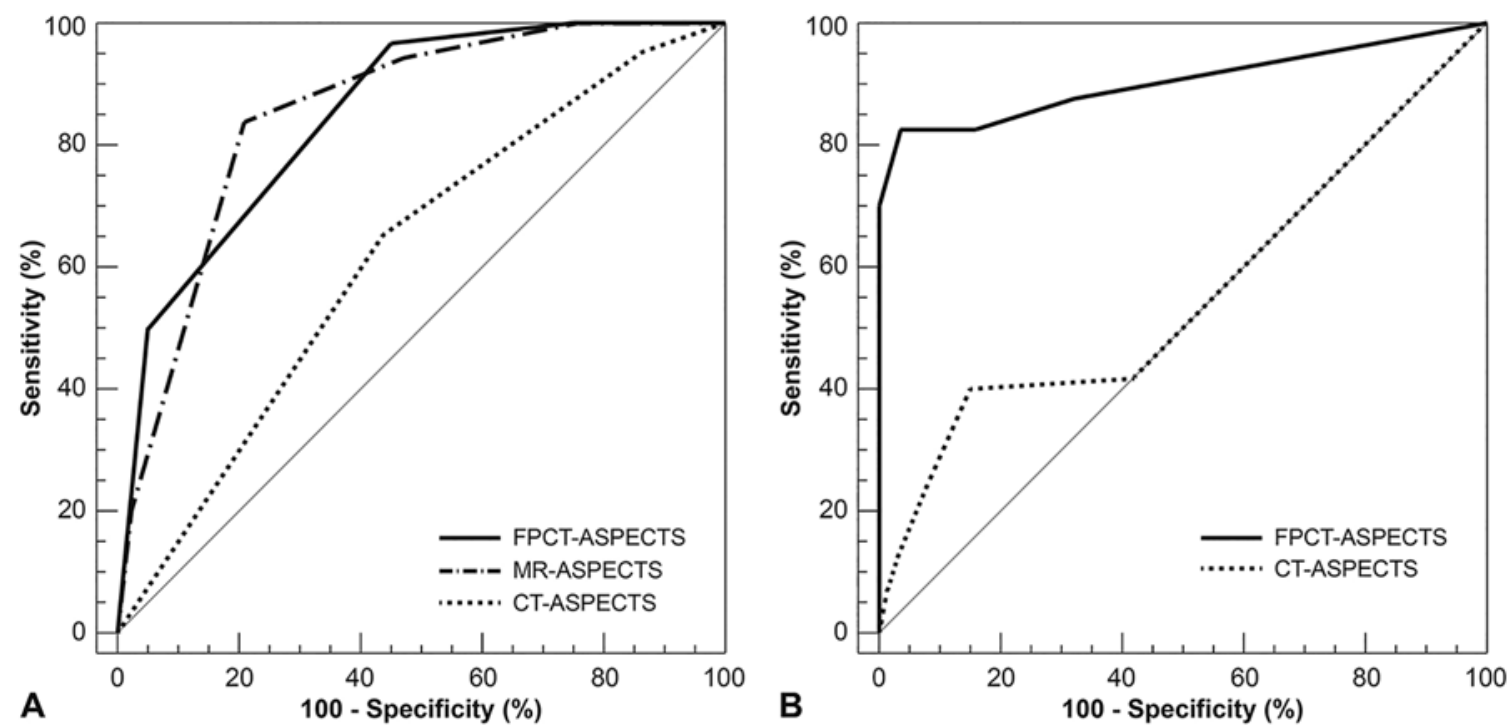

FIG. 3. ROC curves for a favorable outcome and malignant infarction. A: ROC curves of the FPCT-ASPECTS, MR-ASPECTS, and initial noncontrast CT (CT-ASPECTS) for a favorable outcome. The AUC of the FPCT-ASPECTS was 0.862 , which was significantly greater than that of the CT-ASPECTS $(0.637)(p<0.001)$. The AUC of the FPCT-ASPECTS was comparable to that of the MR-ASPECTS $(0.853 ; p=0.983)$. B: ROC curves of the FPCT-ASPECTS and CT-ASPECTS for malignant infarction. The AUC of the FPCT-ASPECTS $(0.906)$ was significantly greater than that of the CT-ASPECTS $(0.552)(p=0.001)$.

up patency of the recanalized artery. In addition to the information about intra- or postprocedural hemorrhage, FPCT might be clinically referred to predict a patient's clinical outcome in advance to obtain MR images after EVT.

Although the ASPECTS on initial CT images (CTASPECTS) is an important prognostic factor for a patient's functional outcome, the CT-ASPECTS was less predictive than the FPCT-ASPECTS in our study. First, the CT-ASPECTS was originally devised to aid in determining treatment eligibility, not for assessing clinical outcome. Second, the CT-ASPECTS only reflects the extent of the infarct core before EVT. Because the infarct core progresses until recanalization is achieved, the final infarct core might be significantly different from the initial infarct core, depending on the status of the collateral vessels and the elapsed time from initial CT to recanalization. For a patient with an initially large infarct core, the CT-ASPECTS might be predictive of a worse clinical outcome. However, the CT-ASPECTS might not predict a good clinical outcome even if a patient has an initially small infarct core. On the contrary, the FPCT-ASPECTS appears to reflect the volume of the final infarct core, as demonstrated by an AUC that is comparable to that of the follow-up MR-ASPECTS.

TABLE 2. Comparison of variables and FPCT-ASPECTS between patients with and those without malignant infarction

\begin{tabular}{|c|c|c|c|c|c|c|}
\hline & \multicolumn{4}{|c|}{ Univariable Analysis } & \multicolumn{2}{|c|}{ Multivariable Analysis } \\
\hline & All Patients $(n=235)$ & Malignant Infarction $(n=17)$ & No Malignant Infarction $(n=218)$ & $p$ Value & OR $(95 \% \mathrm{Cl})^{*}$ & p Value \\
\hline Age, yrs & $68.1 \pm 13.6$ & $66.7 \pm 12.3$ & $68.2 \pm 13.7$ & 0.675 & & \\
\hline Male sex & $118(50.2)$ & $10(58.8)$ & $108(49.5)$ & 0.616 & & \\
\hline Hypertension & $131(55.7)$ & $8(47.1)$ & $123(56.4)$ & 0.461 & & \\
\hline Diabetes & $43(18.3)$ & $1(5.9)$ & $42(19.3)$ & 0.324 & & \\
\hline Smoking & $28(11.9)$ & $2(11.8)$ & $26(11.9)$ & 0.999 & & \\
\hline Atrial fibrillation & $95(40.4)$ & $6(35.3)$ & $89(40.8)$ & 0.799 & & \\
\hline Coronary artery disease & $28(11.9)$ & $2(11.8)$ & $26(11.9)$ & 0.999 & & \\
\hline Initial NIHSS score & $15.0[7.0,23.0]$ & $14.0[4.0,24.0]$ & $15.0[7.0,23.0]$ & 0.019 & $1.03(0.88-1.21)$ & 0.695 \\
\hline Intravenous tPA & $107(45.5)$ & $9(52.9)$ & $98(45.0)$ & 0.616 & & \\
\hline ICA occlusion & $91(38.7)$ & $11(64.7)$ & $80(36.7)$ & 0.036 & $2.07(0.41-10.5)$ & 0.379 \\
\hline CT-ASPECTS & $8.9 \pm 1.3$ & $3.4 \pm 2.9$ & $9.3 \pm 1.1$ & 0.028 & $1.02(0.65-1.59)$ & 0.932 \\
\hline FPCT-ASPECTS & $9.0 \pm 2.0$ & $3.4 \pm 2.9$ & $8.8 \pm 1.4$ & $<0.001$ & $0.42(0.31-0.57)$ & $<0.001$ \\
\hline
\end{tabular}

Univariate results are expressed as mean $\pm \mathrm{SD}$, the number of patients $(\%)$, or median [IQR].

* Odds ratio for malignant infarction. 


\section{Limitations}

Contrast material-related hyperattenuation (contrast staining) can be categorized as contrast extravasation or contrast enhancement. ${ }^{5}$ Compared with contrast extravasation, contrast enhancement on postprocedural CT is known to be less hyperattenuating, is confined to an anatomical border without mass effect, and disappears on follow-up images around 24 hours. Contrast enhancement was not associated with hemorrhagic lesions, whereas contrast extravasation was a negative prognostic sign and highly associated with intracerebral hemorrhage. However, we did not discriminate between contrast extravasation and contrast enhancement for this study. First, for earlier prediction of a patient's clinical outcomes, we wanted to establish the ability of FPCT to predict outcomes right after the EVT procedure, when contrast extravasation and contrast enhancement cannot be distinguished reliably. Second, as our hypothesis was based on the pathomechanism of blood-brain barrier disruption by ischemic injury, the principal observation in this study should include both contrast extravasation and contrast enhancement. The two types of contrast staining might simply reflect the severity of blood-brain barrier damage rather than a completely different pathomechanism. ${ }^{5,20}$ Considering that iatrogenic intracranial hemorrhage due to vessel rupture is very rare during the EVT procedure, hyperattenuation on FPCT, regardless of its severity, should develop in the area of ischemic injury to the blood-brain barrier. In fact, mingled (or undiscriminating) hyperattenuation on postprocedural CT was shown to be significantly associated with final infarct volume in previous studies. ${ }^{2,13,17,18}$ Therefore, as our and previous studies have shown, hyperattenuation on FPCT appears to reflect the final infarct volume.

This study was retrospectively conducted in a single stroke center. Although FPCT has been recommended for patients who undergo EVT at our institution, its performance was not forced under a specific protocol. Thus, FPCT was not performed in all patients who underwent EVT. However, the omission of FPCT was mostly accidental rather than intentional. Fortunately, basic demographics and clinical outcomes were not significantly different between patients with FPCT and those without FPCT (Supplemental Table). Furthermore, our goal was to evaluate the ability of FPCT to predict patients' clinical outcomes, not to determine clinical outcomes according to FPCT's performance. Thus, the fact that FPCT was not performed in all patients is unlikely to have significantly affected our findings.

In addition, the clinical significance of cutoff points of the FPCT-ASPECTS for clinical outcomes might be a little bit limited. Although these points were calculated under statistical significance, an internal or external validation process was not performed in this study. Further validation study is necessary in a prospective or multicenter-based cohort.

\section{Conclusions}

The FPCT-ASPECTS was highly predictive of a favorable outcome and malignant infarction in patients with a successful recanalization by EVT. In addition to the com- mon use of FPCT to evaluate hemorrhagic conditions immediately after EVT, FPCT can help immediately predict clinical outcomes and thereby aid in acute management after EVT.

\section{Acknowledgments}

This research was supported by a grant from the Korea Health Technology R\&D Project through the Korea Health Industry Development Institute (KHIDI), funded by the Ministry of Health and Welfare, Republic of Korea (HC15C1056).

\section{References}

1. Amans MR, Cooke DL, Vella M, et al. Contrast staining on CT after DSA in ischemic stroke patients progresses to infarction and rarely hemorrhages. Interv Neuroradiol. 2014; 20(1):106-115.

2. Antonucci MU, Mocco J, Bennett JA. New insight into transient contrast enhancement on computed tomography after endovascular treatment of stroke. Interv Neuroradiol. 2012; 18(3):303-308.

3. Nakano S, Iseda T, Kawano H, et al. Parenchymal hyperdensity on computed tomography after intra-arterial reperfusion therapy for acute middle cerebral artery occlusion: incidence and clinical significance. Stroke. 2001;32(9):2042-2048.

4. Wildenhain SL, Jungreis CA, Barr J, et al. CT after intracranial intraarterial thrombolysis for acute stroke. AJNR Am J Neuroradiol. 1994;15(3):487-492.

5. Yoon W, Seo JJ, Kim JK, et al. Contrast enhancement and contrast extravasation on computed tomography after intraarterial thrombolysis in patients with acute ischemic stroke. Stroke. 2004;35(4):876-881.

6. Kim BM, Baek JH, Heo JH, et al. Collateral status affects the onset-to-reperfusion time window for good outcome. J Neurol Neurosurg Psychiatry. 2018;89(9):903-909.

7. Pexman JH, Barber PA, Hill MD, et al. Use of the Alberta Stroke Program Early CT Score (ASPECTS) for assessing CT scans in patients with acute stroke. AJNR Am J Neuroradiol. 2001;22(8):1534-1542.

8. Kim BJ, Chung JW, Park HK, et al. CT angiography of collateral vessels and outcomes in endovascular-treated acute ischemic stroke patients. J Clin Neurol. 2017;13(2):121-128.

9. Saver JL, Goyal M, van der Lugt A, et al. Time to treatment with endovascular thrombectomy and outcomes from ischemic stroke: a meta-analysis. JAMA. 2016;316(12):1279-1288.

10. Sheth SA, Sanossian N, Hao Q, et al. Collateral flow as causative of good outcomes in endovascular stroke therapy. $J$ Neurointerv Surg. 2016;8(1):2-7.

11. Yoo AJ, Zaidat OO, Chaudhry ZA, et al. Impact of pretreatment noncontrast CT Alberta Stroke Program Early CT Score on clinical outcome after intra-arterial stroke therapy. Stroke. 2014;45(3):746-751.

12. Kim JT, Heo SH, Cho BH, et al. Hyperdensity on non-contrast CT immediately after intra-arterial revascularization. $J$ Neurol. 2012;259(5):936-943.

13. Lummel N, Schulte-Altedorneburg G, Bernau C, et al. Hyperattenuated intracerebral lesions after mechanical recanalization in acute stroke. AJNR Am J Neuroradiol. 2014;35(2):345-351.

14. Parrilla G, García-Villalba B, Espinosa de Rueda M, et al. Hemorrhage/contrast staining areas after mechanical intraarterial thrombectomy in acute ischemic stroke: imaging findings and clinical significance. AJNR Am J Neuroradiol. 2012;33(9):1791-1796.

15. Payabvash S, Khan AA, Qureshi MH, et al. Detection of intraparenchymal hemorrhage after endovascular therapy in patients with acute ischemic stroke using immediate postprocedural flat-panel computed tomography scan. J Neuroimaging. 2016;26(2):213-218. 
16. Psychogios MN, Buhk JH, Schramm P, et al. Feasibility of angiographic CT in peri-interventional diagnostic imaging: a comparative study with multidetector CT. AJNR Am J Neuroradiol. 2010;31(7):1226-1231.

17. Rouchaud A, Pistocchi S, Blanc R, et al. Predictive value of flat-panel CT for haemorrhagic transformations in patients with acute stroke treated with thrombectomy. J Neurointerv Surg. 2014;6(2):139-143.

18. Schneider T, Mahraun T, Schroeder J, et al. Intraparenchymal hyperattenuations on flat-panel CT directly after mechanical thrombectomy are restricted to the initial infarct core on diffusion-weighted imaging. Clin Neuroradiol. 2018;28(1): 91-97.

19. Dekeyzer S, Reich A, Othman AE, et al. Infarct fogging on immediate postinterventional CT-a not infrequent occurrence. Neuroradiology. 2017;59(9):853-859.

20. Hamann GF, Okada Y, del Zoppo GJ. Hemorrhagic transformation and microvascular integrity during focal cerebral ischemia/reperfusion. J Cereb Blood Flow Metab. 1996;16(6): $1373-1378$.

\section{Disclosures}

The authors report no conflict of interest concerning the materials or methods used in this study or the findings specified in this paper.

\section{Author Contributions}

Conception and design: BM Kim. Acquisition of data: all authors. Analysis and interpretation of data: all authors. Drafting the article: BM Kim, Baek. Critically revising the article: all authors. Reviewed submitted version of manuscript: all authors. Approved the final version of the manuscript on behalf of all authors: BM Kim. Statistical analysis: BM Kim, Baek. Study supervision: BM Kim.

\section{Supplemental Information}

\section{Online-Only Content}

Supplemental material is available with the online version of the article.

Supplemental Table. https://thejns.org/doi/suppl/10.3171/ 2020.7.JNS193214.

\section{Correspondence}

Byung Moon Kim: Yonsei University College of Medicine, Seoul, Republic of Korea. bmoon21@hanmail.net. 